\title{
Neumopatía crónica secundaria al trastorno de la deglución en un paciente con miopatía mitocondrial
}

\author{
Silvia Palacio-Petri' ${ }^{1}$, Olga Lucía Morales-Múnera', Blair Ortiz-Giraldo²
}

\section{RESUMEN}

Introducción: la enfermedad pulmonar crónica secundaria a la đisfagia es una complicación frecuente en los pacientes con enfermedades neuromusculares. Las miopatías mitocondriales son un conjunto de enfermedades que pueden conducir a daño pulmonar progresivo, secundario al síndrome aspirativo crónico.

Caso clínico: niño de 7 años con signos clínicos y rađiológicos de enfermedad pulmonar crónica; además, con desnutrición crónica, debilidad muscular, disfonía y oculoparesia externa crónica multiplanar. Su padre tuvo síntomas similares desde la infancia y requirió alimentación con dieta espesa por trastorno de la deglución.

Se confirma en el paciente la presencia de disfagia como la causa de la neumopatía crónica y se sospecha miopatía congénita hereditaria.

En consecuencia, se realiza el diagnóstico de enfermedad mitocondrial con oculoparesia externa crónica, mediante la secuenciación del gen polimerasa gamma del ADN mitocondrial (POLG).

Conclusiones: en los pacientes con neumopatía crónica se deben considerar las enfermedades neuromusculares en el diagnóstico diferencial. La miopatía mitocondrial con oculoparesia externa crónica progresiva, se asocia con trastorno de la deglución hasta en un 50 \% de los casos. El diagnóstico temprano es importante para retardar el deterioro de la función pulmonar.

\section{PALABRAS CLAVE}

Deglución; Disfagia; Enfermedades Mitocondriales; Enfermedades PuImonares; Miopatías Mitocondriales

\footnotetext{
1 Neumóloga Pediatra, Docente Universidad de Antioquia, Hospital Universitario San Vicente Fundación, Medellín, Colombia.
}

2 Neurólogo Pediatra, Docente Universidad de Antioquia, Hospital Universitario San Vicente Fundación, Medellín, Colombia.

Correspondencia: Silvia Palacio Petri; salomax@yahoo.com

Recibido: octubre 16 de 2018

Aceptado: enero 25 de 2019

Cómo citar: Palacio-Petri S, Morales-Múnera 0L, Ortiz-Giraldo B. Neumopatía crónica secundaria al trastorno de la deglución en un paciente con miopatía mitocondrial. latreia. 2019 Oct-Dic;32(4):321-327. D0I 10.17533/udea.iatreia.26. 


\section{SUMMARY}

Chronic pneumopathy secondary to swallowing disorder in a patient with mitochondrial myopathy

Introduction: Chronic lung disease secondary to dysphagia is a frequent complication in patients with neuromuscular diseases. Mitochondrial myopathies could lead to progressive lung damage due to chronic aspiration syndrome.

Clinical case: Seven-year-old male with clinical and radiological signs of chronic lung disease, as well as low weight, weakness, dysphonia and multiplanar external oculoparesis. His father had similar symptoms during infancy and needed thickened liquid diet due to swallowing disorder. Dysphagia was confirmed as the cause of chronic lung disease and, therefore, hereditary congenital myopathy was suspected. Mitochondrial disease with chronic external oculoparesis was confirmed by molecular sequencing of the mitochondrial DNA gamma polymerase gene (POLG).

Conclusion: Neuromuscular disorders may cause chronic lung disease. Mitochondrial myopathy with progressive chronic external oculoparesis is associated with swallowing disorder in $50 \%$ of the cases. Early diagnosis is important to slow decline in lung function.

\section{KEY WORDS}

Deglutition; Deglutition Disorders; Lung Diseases; Mitochondrial Diseases; Mitochondrial Myopathies

\section{INTRODUCCIÓN}

En los pacientes con neumopatía crónica, sin origen claro, se debe sospechar aspiración crónica silente a la vía aérea, lo cual es una complicación de muchas enfermedades neuromusculares como las miopatías ${ }^{(1-5)}$.

Las miopatías mitocondriales son entidades poco frecuentes, con una prevalencia aproximada de 1 en 10.000 individuos vivos. Se presentan en cualquier edad, aunque los fenotipos más graves se expresan en los primeros años de vida ${ }^{(6)}$. Las enfermedades mitocondriales más conocidas son el síndrome de KearnsSayre (SKS, por sus siglas en inglés), la oftalmoplejía externa crónica progresiva (por sus siglas en inglés, CPEO), la epilepsia mioclónica con fibras rojas rasgadas (por sus siglas en inglés, MERRF), la miopatía mitocondrial, y la combinación de encefalopatía, acidosis láctica y accidente cerebrovascular (por sus siglas en inglés, MELAS) ${ }^{(7)}$.

\section{REPORTE DE CASO}

Niño con 7 años de edad, hospitalizado por cuadro clínico caracterizado por astenia, adinamia, hiporexia, fiebre y dificultad respiratoria. La radiografía del tórax mostró la pérdida de volumen del pulmón izquierdo con opacidad basal ipsilateral (Figura 1).

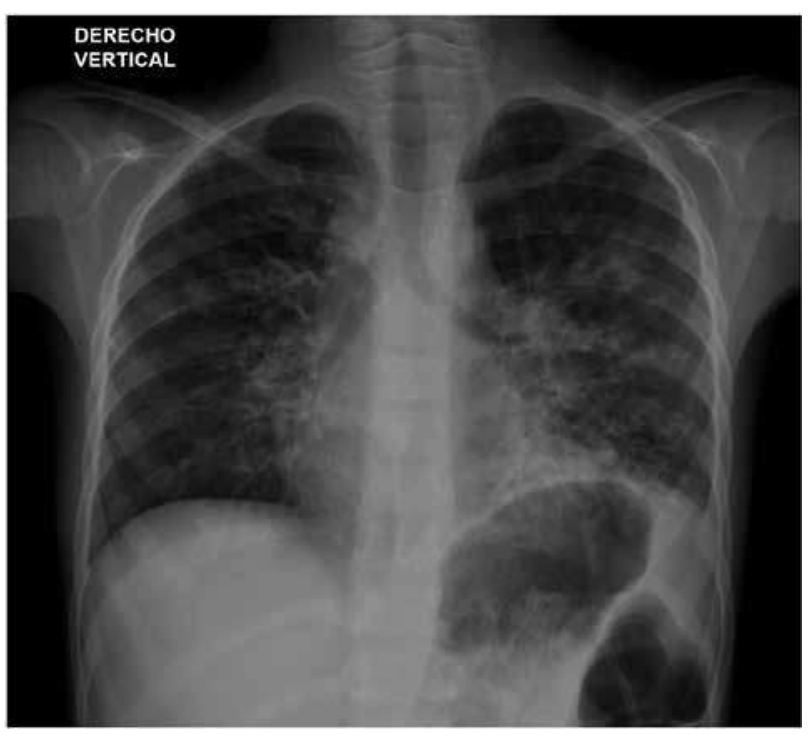

Figura 1. Radiografía de tórax. Evidencia el atrapamiento del aire con pérdida del volumen del pulmón izquierdo, opacidad basal izquierda e imágenes compatibles con bronquiectasias en ambos campos pulmonares. Fuente: propia, con autorización de los padres del paciente para su publicación

Tenía desnutrición aguda grave (Índice masa corporal/edad $-3,35 \mathrm{DE}$, Talla/Edad $-0,52 \mathrm{DE}$ ) y evitaba las actividades que requerían esfuerzo.

Sin antecedentes perinatales de relevancia, presentó hipotonía del lactante y un posible trastorno de la deglución. Había tenido múltiples consultas al servicio 
de urgencias por reagudizaciones respiratorias y un ingreso en la unidad de cuidados intensivos pediátricos por neumonía.

No había consanguinidad entre los padres. El padre tenía antecedente de disfagia y requirió alimentación por sonda en la infancia, posteriormente, se modificó la consistencia de la dieta a una de tipo espeso. Además, presentó debilidad muscular oculofacial faríngea y disfonía, la cual persiste en la actualidad (Figura 2).

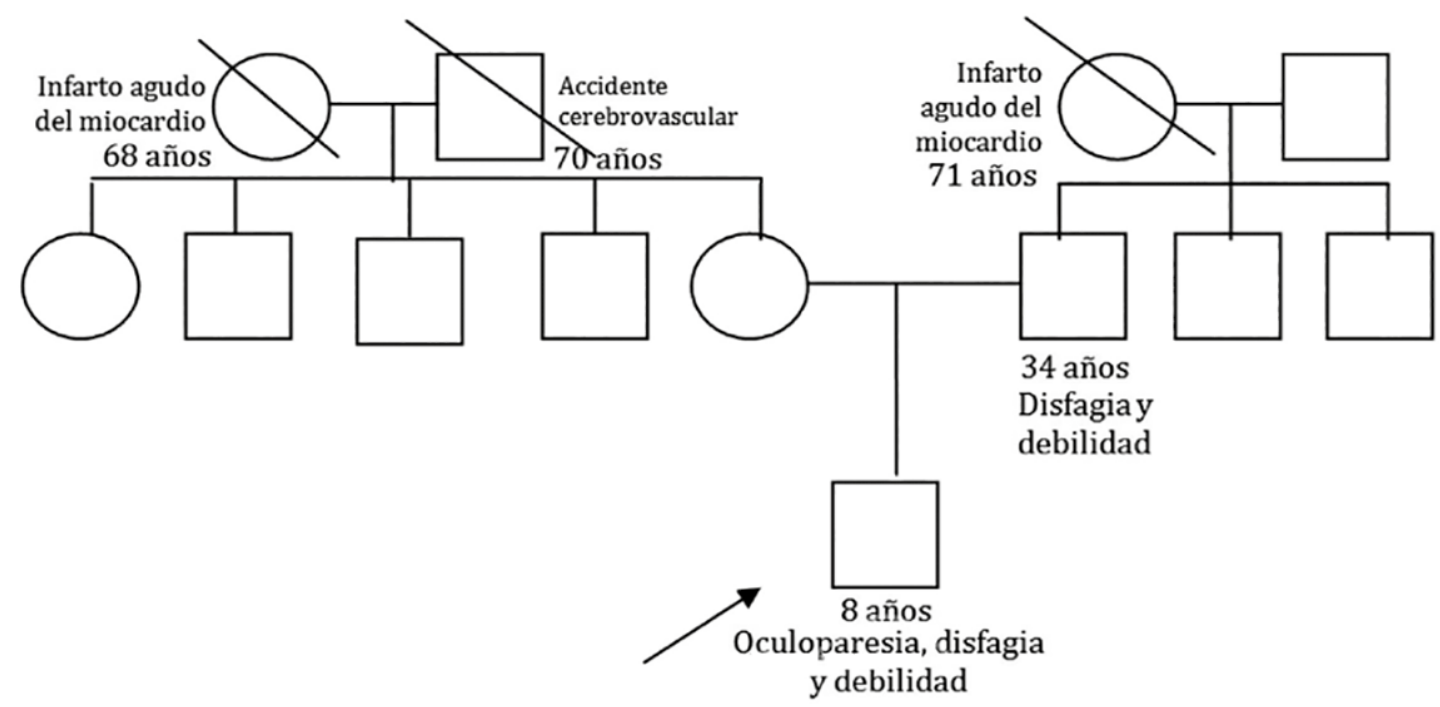

Figura 2. Heredograma. Muestra relación familiar en el mecanismo de transmisión genética de la enfermedad. Fuente: elaboración propia

Al momento del examen físico se encontró un niño enflaquecido, con disminución del diámetro anteroposterior del tórax, crépitos finos, sibilancias y roncus difusos en ambos campos pulmonares, con acropaquia incipiente. Por la sospecha de neumopatía crónica se solicitó tomografía de tórax de alta resolución, que mostró la pérdida de volumen del pulmón izquierdo y múltiples bronquiectasias (Figura 3), espirometría pre y posbroncodilatador con alteración restrictiva moderada. La ecocardiografía, estudios para tuberculosis, fibrosis quística e inmunodeficiencias primarias y secundarias fueron negativos. La cine radiografía de la deglución reveló el trastorno de la deglución severo en la fase faríngea y los episodios de bronco aspiración silente.

El examen neurológico encontró oculoparesia externa multiplanar, especialmente para la supraversión vertical, disminución de los movimientos sacádicos, presencia de reflejo optocinético, paresia del orbicular ocular y labial en forma bilateral sin paresia

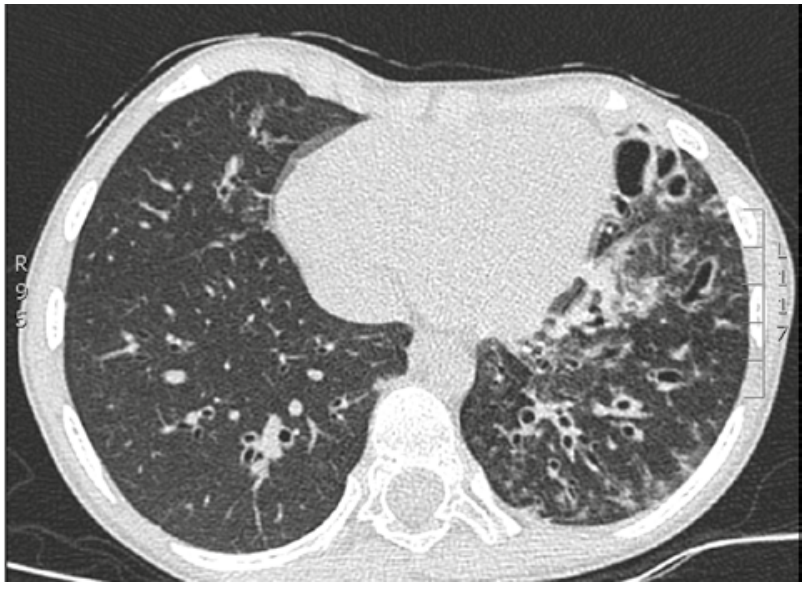

Figura 3. Tomografía de alta resolución de tórax. Se observa la presencia de cambios fibróticos subpleurales apicales bilaterales de predominio derecho, patrón en vidrio esmerilado hacia regiones parahiliares bilaterales de predominio izquierdo, múltiples bronquiectasias, atrapamiento del aire y pérdida de volumen del pulmón izquierdo. Fuente: propia, con autorización de los padres del paciente para su publicación 
lingual, con disfonía y $\nabla 0 z$ nasalizada, hipotrofia e hipotonía muscular de distribución axial y cefaloparesia leve con incapacidad para tocarse el pecho con el mentón. Se encontró hiporrefléxico, con fuerza $4 / 5$ para flexión de hombros, flexión de cadera y extensión de la rodilla (según la Escala de fuerza muscular modificada del Medical Research Council (MRC) y el Signo de Gowers negativo.

Se sospechó miopatía congénita hereditaria, tipo enfermedad mitocondrial con oftalmoplejía crónica, síndrome miasténico congénito o enfermedad de Niemann-Pick tipo C. La electromiografía confirmó la enfermedad intrínseca de la fibra muscular en grupos musculares axiales. Se descartó patología de la unión neuromuscular mediante la prueba de estimulación repetitiva de 2 a $20 \mathrm{~Hz}$ y la enfermedad de Niemann-Pick tipo $C$ mediante la determinación del valor normal del biomarcador lyso-SM-509. Se solicitó la secuenciación Sanger del gen POLG que mostró la variante alélica patogénica p.Ala467Thr, en homocigosis, lo que confirma miopatía mitocondrial con oftalmoplejía externa crónica progresiva.

Dada la gravedad del trastorno de la deglución, la extensión, irreversibilidad y progresión del daño pulmonar, se realizó una gastrostomía enđoscópica percutánea. Además, se formuló coenzima Q10, creatina monohidrato, terapias de fortalecimiento de la musculatura, técnicas de alimentación boca para consistencias seguras y observación expectante de la progresión del trastorno de deglución.

\section{DISCUSIÓN}

EI proceso de la deglución depende de muchos músculos y acciones neurales reflejas. Se inicia voluntariamente, pero a partir de la fase faríngea continúa bajo el control involuntario ${ }^{(8)}$.

El síndrome aspirativo es la principal causa de la enfermedad pulmonar crónica en pacientes con la enfermedad neuromuscular, causando daño estructural del parénquima pulmonar, enfermedad de la vía aérea $y$ bronquiectasias ${ }^{(4)}$, hallazgos presentes en el paciente. Las aspiraciones repetidas producen alteración del reflejo protector de la tos por la sobreestimulación de los receptores, tornándose silentes. Este paciente ingresó por un problema pulmonar agudo con estigmas físicos y radiológicos de enfermedad pulmonar crónica. Estos hallazgos, aunados a que su padre presentaba características clínicas similares, generaron la sospecha de una enfermedad hereditaria.

Se estableció que el paciente tenía miopatía mitocondrial, un grupo heterogéneo de enfermedades que se presentan por alteraciones genéticas, estructurales $y$ bioquímicas mitocondriales.

Debido a que las mitocondrias son la principal fuente de producción de energía, las características clínicas involucran, típicamente, los tejidos con altos requerimientos energéticos, como son el sistema nervioso, el sistema endocrino y los músculos, particularmente los extraoculares, deglutorios y cardíacos ${ }^{(6)}$.

Estas enfermedades son producto de una alteración en la fosforilación oxidativa en la cadena respiratoria que está controlada por el ADN nuclear (ADNn) $y$ mitocondrial (ADNmt). Los defectos mendelianos mitocondriales pueden abarcar subunidades de los complejos de la cadena respiratoria, las proteínas de ensamble mitocondrial, la traducción del ADNmt, composición lipídica de la membrana mitocondrial interna o la dinámica de la misma organela ${ }^{(9)}$. Solo en el $40 \%$ de los adultos y el $10 \%$ de los niños se encuentra alguna de las mutaciones reportadas ${ }^{\left({ }^{6}\right)}$, en nuestro paciente se identificó dicha mutación.

El gen POLG está ubicado en el locus 15q26.1. El gen se encarga de activar la subunidad alfa de la proteína polimerasa gamma (pol $\gamma$ ), que es la única DNA polimerasa mitocondrial. Este gen tiene un papel crítico en la reparación y replicación del DNA mitocondrial (mtDNA). Las enfermedades relacionadas con los cambios genéticos patogénicos incluyen el espectro de ataxia neuropatía, miocerebrohepatopatía de la niñez, epilepsia mioclónica con miopatía y ataxia sensitiva, oftalmoplejía externa progresiva y síndrome de Leigh. Se han reportado, al menos, 67 mutaciones en POLG relacionadas con CPEO, la mayoría corresponden con cambios en un aminoácido en la subunidad alfa de la POLG, que se traduce en una disminución de la eficiencia para replicar el mtDNA. El síndrome de Alpers-Huttenlocher y la CPEO, con frecuencia, son ocasionados por la mutación POLG Ala467Thr. En la actualidad se habla del término «desórdenes relacionados con POLG» ${ }^{(10)}$.

En la miopatía mitocondrial se afecta gravemente la estructura y función del músculo esquelético por el 
malfuncionamiento de la fosforilación oxidativa ${ }^{(7,11)}$. Las fibras musculares tipo 1 se caracterizan por la resistencia a la fatiga y poca fuerza. También son las más afectadas, debido a que contienen abundante cantidad de mitocondrias. Las fibras tipo 2 se afectan en menor medida. En nuestro paciente no se realizó biopsia muscular porque el diagnóstico pudo establecerse a partir de la presunción electroclínica y se confirmó mediante la secuenciación del gen POLG. Actualmente, la biopsia muscular se ha reservado para los pacientes cuyo diagnóstico electroclínico y molecular no es concluyente; en estos casos es fundamental hacer tinción con tricrómico de Gomori, citocromo oxidasa, succinato deshidrogenasa, NADH y microscopía electrónica en el espécimen de patología.

Principalmente, por los hallazgos oculares, el fenotipo del paciente fue específicamente sugestivo de oftalmoplejía externa progresiva crónica (Tabla 1).

La CPEO se caracteriza por ptosis palpebraI, alteración de los movimientos oculares con pupilas normales y, en algunos casos, cursa con afectación de la musculatura facial ${ }^{(12-13)}$. Puede asociarse con un compromiso multisistémico con la afectación de la retina, el cerebelo, el corazón y los músculos laríngeos ${ }^{(13-15)}$. Nuestro paciente presentaba trastorno grave de la deglución con aspiraciones silentes y la alteración de la $\nabla \mathrm{Oz}$, consistente en baja tonalidad y disfonía. Además, presentaba oculoparesia externa multiplanar.

El compromiso respiratorio incluye disminución de la fuerza de los músculos inspiratorios y espiratorios, evidenciado en la disminución de la capacidad vital forzada (CVF), el volumen espiratorio forzado en un segundo (VEF1) y el flujo pico espiratorio (PEF). La debilidad de los músculos respiratorios se correlaciona con la debilidad de la cintura, más no con otras características clínicas como la duración o gravedad de la enfermedad o el tipo de mutación. Estos pacientes son particularmente susceptibles a tener neumonía, dada la presencia concurrente de debilidad laríngea, tos inefectiva por cierre incompleto de la glotis, alteración del tono y la presión del esfínter esofágico superior y alteración del tránsito faríngeo y esofágico, todo lo cual conduce a fenómenos aspirativos. Los trastornos de la deglución se presentan en el 50 \% de Ios pacientes con CPEO ${ }^{(7,14)}$. La neumonía es la principal causa de muerte $y$ puede tener un curso más grave que conlleva a la falla ventilatoria y dificultades en el retiro del soporte ventilatorio. Adicionalmente, los pacientes pueden presentar dificultades en el control de las infecciones ${ }^{(14)}$.

\section{Tabla 1. Características clínicas de la oftalmoplejía externa progresiva crónica por mutación de POLG}

\begin{tabular}{cc|}
\hline $\begin{array}{c}\text { Características clínicas } \\
\text { de la oftalmoplejía externa progresiva } \\
\text { crónica por mutación POLG (15) }\end{array}$ & $\begin{array}{c}\text { Encontrado } \\
\text { en el } \\
\text { paciente }\end{array}$ \\
Debilidad muscular & $\mathrm{X}$ \\
Cataratas & \\
\hline Sordera de cualquier tipo & \\
\hline Deuropatía sensitiva axonal & \\
Hipogonadismo & \\
Parkinsonismo & \\
Ataxia cerebelosa & \\
Pie cavo & \\
Temblor & \\
\hline Discapacidad intelectual & \\
\hline Rabdomiólisis por alcohol & \\
Oftalmoplejía & \\
\hline Antecedentes familiares de CPEO \\
Lactoacidosis \\
\hline Intolerancia al ejercicio \\
Retinopatía pigmentaria \\
\hline
\end{tabular}

Fuente: elaboración propia

La CPEO se manifiesta con síntomas leves en la niñez, tal como ocurrió en el paciente que reportamos (16). La debilidad muscular ocular se desarrolla gradualmente durante años e incluso décadas. Puede ocurrir por mutación esporádica (deleción única), herencia mitocondrial (matrilínea) o ser autosómica dominante o recesiva ${ }^{(7)}$.

Algunas mutaciones de POLG se han encontrado con efecto tanto dominante como recesivo, y la variabilidad clínica para homocigotos es muy variable. Se ha sugerido que las manifestaciones clínicas se ven influenciadas por factores modificadores como la composición del ADN mitocondrial, otros genes nucleares, factores epigenéticos y factores ambientales. Aquí se encontró en el paciente reportado la mutación 
p.Ala467Thr en estado de homocigosis, sugiriendo una herencia autosómica recesiva. Aunque los padres no tenían la secuenciación para el gen en cuestión, si la madre y el padre fueran portadores de la mutación, la mutación tendría un comportamiento recesivo. Otra posibilidad es que la madre sea portadora y el padre homocigoto, caso en el cual, la mutación también se heredaría en forma autosómica recesiva. Esta situación ilustra la complejidad en la herencia de esta patología, y justifica la necesidad de la evaluación molecular de los padres y la valoración por genetista para una consejería genética adecuada ${ }^{(10)}$.

Los ensayos terapéuticos realizados hasta el momento no han sido exitosos. Dos grandes revisiones clínicas no encontraron evidencia que demuestre el beneficio de los agentes terapéuticos o suplementos nutricionales ${ }^{(1,17-18)}$.

\section{CONCLUSIÓN}

La neumopatía crónica aspirativa es una complicación grave de las enfermedades neuromusculares, es imprescindible su reconocimiento temprano e inicio oportuno de intervenciones para retardar el deterioro de la función pulmonar en los pacientes con enfermedades neuromusculares. Ante el hallazgo de neumopatía crónica aspirativa se debe sospechar de la posibilidad de una enfermedad neuromuscular de base, es indispensable un equipo multidisciplinario para el diagnóstico, seguimiento y la asesoría genética.

\section{AGRADECIMIENTOS}

Agradecemos a la familia por permitirnos publicar el caso de este niño.

\section{CONFLICTOS DE INTERESES}

Ninguno por declarar.

\section{FUENTE DE FINANCIACIÓN}

Ninguna.

\section{REFERENCIAS BIBLIOGRÁFICAS}

1. Bacco J, Araya F, Flores E, Peña N. Trastornos de la alimentación y deglución en niños y jóvenes portadores de parálisis cerebral: abordaje multidisciplinario. Rev. méd. Clín. 2014;25(2)330-42. DOI 10.1016/S07168640(14)70044-6.

2. García J. Disfagia en la infancia. An Pediatr Contin. 2014;12(5):221-30. DOI: 10.1016/S16962818(14)70195-7.

3. Henao P, Lopera M, Salazar O, Medina P, Morales O. Guía de práctica clínica basada en la evidencia para el diagnóstico de disfagia en niños. Iatreia. 2009;22(2):169-79.

4. Moënne K. Aporte del estudio por imágenes en el estudio de alteraciones respiratorias en niños con limitaciones neurológicas. Neumol Pediatr. 2011;6(2):75-9.

5. Cámpora H, Falduti A. Evaluación y tratamiento de las alteraciones de la deglución. Rev Am Med Resp. 2012;3:98-107.

6. Klopstock T, Jaksch M, Gasser T. Age and cause of death in mitochondrial diseases. Neurology. 1999;53(4):855-7.

7. Domenis DR, Granzotti RB, Sobreira CF, Dantas RO. Pharyngeal transit in patients with chronic progressive external ophthalmoplegia. Int J Speech Lang Pathol. 2015;17(4):384-9. DOI 10.3109/17549507.2014.941935.

8. Rebolledo F. Alimentación y deglución. Aspectos relacionados con el desarrollo normal. Plast \& Rest Neurol. 2005;4(1-2):49-57.

9. Vincent AE, Rosa HS, Pabis K, Lawless C, Chen C, Grünewald A, et al. Sub-cellular origin of mtDNA deletions in human skeletal muscle. Ann Neurol. $2018 \mathrm{Jul}$ 16;84(2):289-301 . DOI: 10.1002/ana.25288.

10. Neeve V, Samuels D, Bindoff A, Bosch B, Goethem G, Smeets $\mathrm{H}$, et al. What is influencing the phenotype of the common homozygous polymerase- $\gamma$ mutation p.Ala467Thr? Brain. 2012 Dec;135(12):3614-26. DOI 10.1093/brain/aws298.

11. Hsiao CC, Lee NC, Huang PH, Tsai TH. Histopathological and genetic analysis of extraocular muscle in chronic progressive external ophthalmoplegia. J Formos Med Assoc. 2016;115(11):1012-14. DOI 10.1016/j. jfma.2016.04.010. 
12. Ringel SP, Wilson WB, Barden MT, et al. Extraocular muscle biopsy in chronic progressive external ophthalmoplegia. Ann Neurol. 1979;6(4):326-39. DOI 10.1002/ana.410060406.

13. Jiménez-Caballero PE, Serviá M, Cabeza CI, Marsal-Alonso C, Alvarez-Tejerina A, et al. Chronic progressive external ophthalmoplegia: clinical and electromyographic manifestations in a series of cases. Rev Neurol. 2006;43(12):16-31.

14. Smits B, Heijdra Y, Cuppen F, Van Engelen BG. Nature and frequency of respiratory involvement in chronic progressive external ophthalmoplegia. J Neurol. 2011;258(11):2020-5. DOI 10.1007/s00415011-6060-7.

15. Steele HE, Horvath R, Lyon JJ, Chinnery PF. Monitoring clinical progression with mitochondrial disease biomarkers. Brain. 2017 Oct 1;140(10):2530-40. DOI 10.1093/brain/awx168.

16. Gehrig SM, Mihaylova V, Frese S, Mueller SM, LigonAuer M, Spengler CM, et al. Altered skeletal muscle (mitochondrial) properties in patients with mitochondrial DNA single deletion myopathy. Orphanet J Rare Dis. 2016 Jul 29;11(1):105. DOI 10.1186/s13023-016-0488-X.

17. Pfeffer G, Majamaa K, Turnbull DM, Thorburn D, Chinnery PF. Treatment for mitochondrial disorders. Cochrane Database Syst Rev. 2012;(4):CD4426. DOI 10.1002/14651858.CD004426.pub3.

18. Parikh S, Goldstein A, Koenig MK, Scaglia F, Enns GM, Saneto R, et al. Diagnosis and management of mitochondrial disease: a consensus statement from the Mitochondrial Medicine Society. Genet Med. 2015;17(9):689-701. DOI 10.1038/gim.2014.177. 